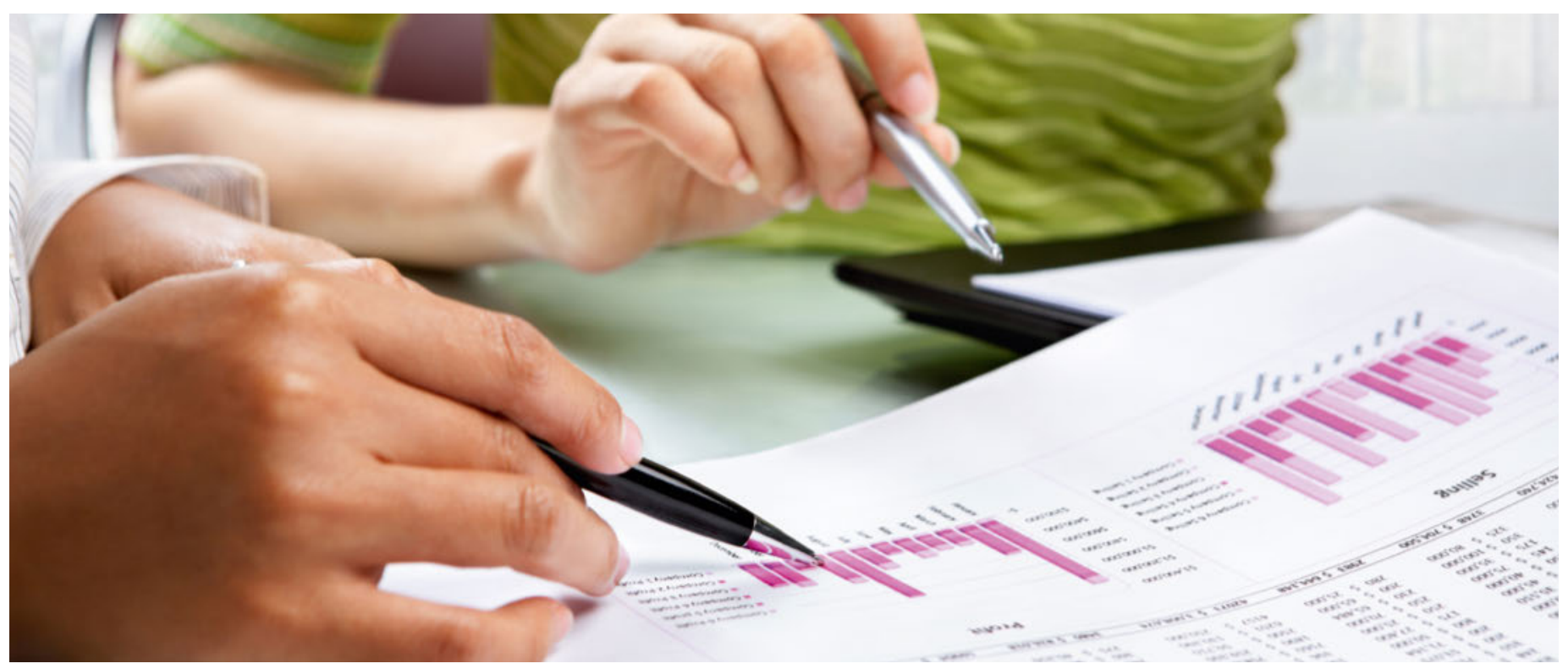

Wirtschaftlichkeitsprüfung - Weiterentwicklung und Überprüfung der statistischen Methode

\title{
Neue Wege bei der Wirtschaftlichkeitsprüfung
}

Thomas Kessler ${ }^{a}$, Mirjam D’Angelo ${ }^{b}$, Anke Trittinc

${ }^{a} \mathrm{FMH}$, Abteilung Ambulante Versorgung und Tarife, Experte; ${ }^{b}$ santésuisse, Projektleiterin Wirtschaftlichkeitsprüfungen; ${ }^{c}$ curafutura, Leiterin Tarife

Seit 2013 verpflichtet das Krankenversicherungsgesetz Versicherer und Leistungserbringer, gemeinsam eine Methode zur Kontrolle der Wirtschaftlichkeit medizinischer Leistungen vertraglich festzulegen. Als Prüfmethode hat sich ein paritätisch zusammengesetztes Projektteam, bestehend aus Vertretern von santésuisse, curafutura und FMH, auf die Varianzanalyse geeinigt, die mit zusätzlichen Morbiditätsfaktoren weiterentwickelt wird. Jetzt haben sich die Vertragsparteien darauf geeinigt, dieses statistische Modell der Wirtschaftlichkeitsprüfung extern validieren zu lassen.

Seit mehr als 30 Jahren prüfen die Krankenversicherer die Wirtschaftlichkeit der ambulant tätigen Ärzteschaft. Einerseits tut dies jeder einzelne Versicherer mittels Einzelrechnungskontrollen, andererseits führt santésuisse im Auftrag der Gesamtbranche mittels statistischer Methoden systematische Wirtschaftlichkeitsprüfungen durch.

\section{Unwirtschaftlichkeit erkennen}

Die statistische Methode hat zum Ziel, diejenigen Leistungserbringer zu erkennen, die beispielsweise mit «Überarztung» (Polypragmasie) - also dem Verstoss gegen das Wirtschaftlichkeitsgebot - signifikant höhere Kosten generieren als ihre Facharztkolleginnen und -kollegen. Dabei werden die Kosten pro Erkrankten mit den durchschnittlichen Kosten der gleichen Facharztgruppe verglichen; die Rede ist vom Durchschnittskostenvergleich. Leistungserbringer mit überdurchschnittlich hohen Kosten werden mittels Informationsschreiben kontaktiert und gebeten, ihr identifiziertes Kostenbild zu begründen. Können die zu hohen Kosten nicht erklärt werden - oder unternimmt der Arzt bei einem unerklärten Kostenbild seiner Praxis nicht genügend Anstrengungen, um dieses zu optimieren -, stellt santésuisse Rückzahlungsforderungen. Dies kann 
in seltenen Fällen zu Gerichtsverfahren führen. Rückzahlungsforderungen sind denn auch nicht das Ziel der Wirtschaftlichkeitsprüfungen, können jedoch die Konsequenz sein.

\section{Morbidität des Erkranktenkollektivs als zusätzlicher Faktor}

Schon vor Inkrafttreten des neuen Gesetzesartikels Anfang 2013 haben FMH und santésuisse in einer Absichtserklärung die gemeinsamen Ziele zur Weiterentwicklung der Wirtschaftlichkeitsprüfung definiert. Neben dem Bekenntnis, dem Auftrag des Gesetzgebers gemeinsam nachzukommen, soll die statistische Methode derart angepasst werden, dass unter Berücksichtigung der Morbidität des Erkrankten-Kollektivs lediglich effektiv unwirtschaftlich arbeitende Ärzte identifiziert werden. Zusätzlich soll die statistische Methode regelmässig überprüft, angepasst und ergänzt werden.

Ziel muss es sein, in Zukunft möglichst wenig Ärzte ungerechtfertigterweise als unwirtschaftlich arbeitend zu identifizieren.

Ein paritätisches Projektteam, bestehend aus Vertretern von santésuisse, curafutura und FMH, nahm sich der Frage nach der geeigneten Methode zur Prüfung der Wirtschaftlichkeit an und erarbeitete Lösungsvorschläge. Daraus resultierte die vertragliche Vereinbarung zur Varianzanalyse (analysis of variances ANOVA). Der Vertrag wurde fristgerecht im Januar 2014 von allen Parteien unterzeichnet.

\section{Weniger ungerechtfertigte Verdächtigungen}

Teil des unterzeichneten Vertrags vom Januar 2014 ist die Weiterentwicklung der festgelegten Methode um zusätzliche Morbiditätsfaktoren. Es herrscht Konsens, dass die Morbidität der Erkrankten massgeblich deren Behandlungskosten beeinflusst. Ärzte mit überdurchschnittlich morbiden Patienten weisen entsprechend höhere Kosten auf. Dies soll inskünftig bereits im statistischen Modell berücksichtigt werden, so dass einerseits weniger Ärzte mit überdurchschnittlich morbidem Erkrankten-Kollektiv ungerechtfertigt als unwirtschaftlich arbeitend verdächtigt werden, was mit Rechtfertigungskosten verbunden ist. Andererseits erscheinen in der Wirtschaftlichkeitsprüfung bis dato nicht auffällige Leistungserbringer mit unterdurchschnittlich morbidem Kollektiv - und entsprechend tieferen jährlichen Behandlungsfallkosten - neu als statistisch auffällig.

\section{Kostentreibende Ursachen berücksichtigen}

Die Morbidität direkt abzubilden ist schwierig; sie ist nur schwer messbar, und schweizweit liegen keine Daten in ausreichender Qualität dazu vor. Es gilt deshalb verschiedene Möglichkeiten zu prüfen, diese in direkt abzubilden. So zum Beispiel mittels Alter der Erkrankten, ihrer Franchise oder über den Medikamentenkonsum. Weitere wichtige Faktoren für das Modell könnten unter anderem auch der Praxisstandort oder das Leistungsangebot des Arztes darstellen. Ziel des statistischen Modells ist es, möglichst alle exogenen Faktoren abzubilden, die zwar hohe Kosten verursachen, jedoch nicht mit einer «Überarztung» gleichzusetzen sind. Konsequenterweise sollen vor allem die äusseren, kostentreibenden Ursachen berücksichtigt werden, die nicht direkt durch das Verhalten des Arztes beeinflussbar sind.

\section{Externe Validierung der statistischen Methode}

Im Herbst 2016 haben sich die Parteien geeinigt, die statistische Methode, das Modell und mögliche Weiterentwicklungen, extern validieren zu lassen. Eine neutrale, auf gesundheitsökonomische Beratungen spezialisierte Stelle soll die Methode prüfen und Empfehlungen für deren Weiterentwicklung liefern bzw. alternative statistische Methoden empfehlen, wenn die bestehende statistische Methode in ihrer Zielerreichung ungenügend wäre. Im Rahmen einer Ausschreibung wurde der Auftrag an das Beratungsunternehmen Polynomics AG vergeben. Dies, nachdem Versicherer und FMH im Rahmen eines Dialogverfahrens detaillierte Offerten mehrerer Anbieter geprüft hatten.

Die externe Validierung soll sicherstellen, dass die statistische Methode aktuellen wissenschaftlichen Standards genügt und wo nötig weitere geeignete exogene Faktoren hinzugezogen werden, um das Modell anzupassen. Zudem wird extern unabhängig überprüft, ob mit der bestehenden statistischen Methode die unwirtschaftlich tätigen Ärzte erkannt werden. Ziel muss es sein, in Zukunft möglichst wenig Ärzte ungerechtfertigterweise als unwirtschaftlich arbeitend zu identifizieren. Dies liegt im Interesse aller Beteiligten, nicht zuletzt, weil der Aufwand für die Wirtschaftlichkeitsprüfung reduziert werden kann. Der Validierungsbericht wird im zweiten Quartal 2017 vorliegen.

Bildnachweis

(c) Otnaydur | Dreamstime.com 